\title{
Funcionamiento familiar y adicción a internet en estudiantes de una institución educativa pública de Lima Norte
}

\author{
Family functioning internet addiction among students of a public school in North Lima
}

Cristian Adriano Rengifo', Luis Mamani Ferrúa², Wildman Vilca Quiro³

\begin{abstract}
RESUMEN
Objetivo: Determinar la relación entre funcionamiento familiar y adicción a internet de los alumnos adolescentes de una institución educativa pública de Lima Norte. Material y métodos: Se utilizó el diseño no experimental de corte transversal y de tipo correlacional. Asimismo, se empleó un muestreo no probabilístico intencional, incluyendo alumnos de ambos sexos con edades entre 12 y 20 años. Resultados: El 44\% de los adolescentes presenta un funcionamiento familiar de rango medio y solo un $21.7 \%$ tiene un funcionamiento extremo. Asimismo, el 86\% de los participantes evidencia un uso normal de internet, el 13\% se encuentran en riesgo de adicción y solamente cerca del $1 \%$ presenta uso adictivo de internet. Además, no se encontró relación significativa entre las variables de estudios (Chi = 4,079; p>,395), a excepción de cohesión familiar $(r=-.223, p<0.01)$ Conclusiones: El funcionamiento familiar no se relaciona significativamente con la adicción a internet. Sin embargo, se encontró relación negativa con cohesión familiar, es decir, cuanto mayor es la cohesión familiar menor es el riesgo de adicción a internet.
\end{abstract}

Palabras clave: Funcionamiento Familiar, Cohesión, Adaptabilidad, Adicción a Internet.

\begin{abstract}
Objetive: To determine the relationship between family functioning and Internet addiction adolescent students from a public school in northern Lima. Methods: non-experimental and cross-sectional design was used correlational. An intentional non-probability sampling, including students of both sexes aged between 12 and 20 years were also used. Results: $44 \%$ of adolescents have a family operation midrange and only $21.7 \%$ have an extreme performance. Also 86\% of participants evidenced a normal Internet use, $13 \%$ are at risk of addiction and only about $1 \%$ are addictive Internet use. Furthermore, there was no significant relationship between the variables of study (Chi $=4.079 ; \mathrm{p}>$.395), except for family cohesion $(r=-.223$, $\mathrm{p}<0.01)$. Conclusions: The family functioning was not significantly related to Internet addiction. However, it was found that a negative relationship with family cohesion; that is the greater family cohesion, the lower the risk of internet addiction.
\end{abstract}

Keywords: Family Functioning, Cohesion, Adaptability, Internet Addiction.

\footnotetext{
${ }^{1}$ Psicólogo del Servicio Educacional Hogar y Salud, Misión Central Oeste del Perú.

${ }^{2}$ Psicólogo, Centro de Atención Psicológica, Universidad Peruana Unión, Lima, Perú.

${ }^{3}$ Docente de EP Psicología, Facultad de Ciencias de la Salud, Universidad Peruana Unión, Lima, Perú.
} 


\section{INTRODUCCIÓN}

En la actualidad la sociedad está inmersa en las nuevas tecnologías de la información, las cuales han ocasionado cambios paulatinos en la vida cotidiana de las personas (Echeburúa, Labrador y Becoña, 2009), aunque no se puede negar las bondades de ellas (Puertas y Carbonell, 2013), tampoco se puede omitir su otra cara, en especial el uso de internet que ha provocado consecuencias negativas por su uso desmedido (Camelo, León y Salcedo, 2013).

Por un lado, las estadísticas mundiales son reveladoras en relación al incremento acelerado de usuarios a internet, siendo el continente asiático donde existen mayores índices de penetración (Corea del Sur, 82.5\%; Japón, 79.5\%), no obstante la realidad latinoamericana indica cifras considerables tales como en Argentina 66.4\% y Chile 58.6\% (Éxito Exportador, 2012). Asimismo el Perú ha denotado en los últimos años un crecimiento de $36.0 \%$ en el 2011 y 38,2\% en el 2012 (Banco Mundial, 2014). Cabe resaltar que hasta la fecha estas cifras pudieran haberse incrementado por la rápida extensión que viene ejerciendo la red.

Con el incremento del internet han aparecido problemas con su uso. Diversas estadísticas muestran a Estados Unidos y España con los más altos niveles de dependencia a la web, $41 \%$ y $48 \%$ respectivamente (Universia, 2010). En Argentina el 17.1\% de personas de 13 a 30 años presentaba uso abusivo de internet y 4\% uso patológico (Luque, 2009). En muchos casos esta adicción o uso compulsivo ha provocado graves consecuencias, tales como la muerte de jóvenes por agotamiento, después de pasar varias horas jugando en línea sin parar (La Prensa, 2014). En el Perú el Ministerio de Salud (MINSA) informó que el 2012 atendió a 190 adolescentes entre 12 y 17 años con problemas de adicción a internet, y en el 2013 este número se elevó a 201 casos (Perú 21, 2014).

La adolescencia es la etapa donde existe mayor riesgo a adquirir adicción a internet, debido a los cambios, la confusión, consolidación de la identidad personal y sentimientos de frustración experimentados; a esto se añade frecuentemente el consumo de alcohol, tabaco u otras sustancias como una forma de atenuar la ansiedad, inseguridad o profundos vacíos internos, así como la necesidad de establecer las primeras relaciones íntimas y amorosas, siendo precisamente el internet quien proporciona esas bondades (Echeburúa et al. 2009; Morales, 2013).

Evidentemente ante todo lo mencionado queda claro que se está ante una realidad problemática: la adicción a internet. Independientemente de la falta de consenso con respecto a este fenómeno, las investigaciones revelan un problema con respecto al uso compulsivo cuyos síntomas son parecidos a la adicción de una sustancia (Echeburúa et al. 2009). Young (1998b) describe este fenómeno como un deterioro en el control del uso de la red, manifestado en diversos síntomas cognitivos, conductuales y fisiológicos.

Frente a esta realidad es necesario identificar los factores y determinantes de la adicción a internet. Echeburúa et al. (2009) señalan dos factores psicológicos que predisponen a este tipo de adicción. El primero se refiere a variables de personalidad tales como la impulsividad, buscar sensaciones, autoestima baja, intolerancia a los estímulos displacenteros y estilo de afrontamiento inadecuado a las dificultades. El segundo hace referencia a la vulnerabilidad emocional, manifestando estado de ánimo disfórico, carencia de afecto, pobreza de relaciones sociales y cohesión familiar débil. Asimismo Muñoz-Rivas, Redondo, Fernández y Gámez- Gaudix (2011) afirman la participación de variables individuales, familiares y sociales como factores de riesgo asociados a la adicción a internet.

Ambos autores ponen en relevancia el importante papel que estaría jugando la familia en relación a este fenómeno psicológico. Siendo que es la principal responsable del cuidado y la protección de los niños, desde la infancia a la adolescencia (Vallejo y Capa, 2010). Entendiéndose que una dinámica disfuncional en la familia contribuiría a la utilización peligrosa de internet por parte del adolescente (Toro, 2010).

Olson (citado por Polaino-Lorente y Martínez, 2003) define el funcionamiento familiar como la interacción dinámica y sistémica de dos dimensiones principales de la vida familiar: cohesión y adaptabilidad familiar. Donde lo funcional se daría en familias que muestran niveles moderados en ambas dimensiones, y lo disfuncional en familias que alcanzan niveles extremos. La cohesión es considerada como el vínculo emocional que mantienen los miembros de la familia y el nivel de autonomía individual que una persona experimenta en su contexto familiar; por su parte la adaptabilidad tiene que ver con la medida en que el sistema familiar es flexible y capaz de cambiar su estructura de poder, sus roles y reglas de relación en respuesta a una demanda situacional o de desarrollo (Polaino-Lorente y Martínez, 2003; Arenas, 2009).

Cruzado, Matos y Kendall (2006) en un estudio realizado en Lima con pacientes adictos a internet encontraron una historia de disfunción familiar en el $80 \%$ de los pacientes; haciendo ver que la falta de soporte familiar es uno de los factores que predisponen 
al uso problemático de internet. Por su parte Zapata (2013) en un estudio realizado en adolescentes atendidos en consulta externa del Hospital Hermilio Valdizán encontró que el 57.4\% provenía de una familia disfuncional. Asimismo Matalinares et al. (2013) aseveran que los estilos parentales disfuncionales (abuso e indiferencia) influyen en la adicción al Internet.

Frente a estos alcances no se puede negar el papel explícito que tiene la familia para que los adolescentes y/o jóvenes puedan desarrollar conductas adictivas a internet. Vallejo y Capa (2010) afirman que el factor explicativo más importante de la adicción a internet es la funcionalidad familiar.

Por ello, el objetivo de esta investigación es determinar si existe relación significativa entre funcionamiento familiar y adicción a internet en adolescentes de una institución educativa pública de Lima norte.

\section{MATERIAL Y MÉTODOS}

Para llevar a cabo la investigación se utilizó el diseño no experimental de corte transversal y de tipo correlacional, siendo que la naturaleza de la investigación es relacionar las variables de estudio (Fontes et al., 2010).

\section{Participantes}

Participaron 225 alumnos de una institución educativa pública de Lima-norte, que se encontraban cursando entre el primero y quinto grado de secundaria, seleccionados a través de un muestreo no probabilístico intencional. Se incluyó a adolescentes de ambos sexos con edades entre 12 y 20 años, quienes completaron los cuestionarios de forma voluntaria.

\section{Instrumentos}

Los componentes del cuestionario incluyeron una declaración de consentimiento informado, un cuestionario de elaboración propia (datos sociodemográficos e información sobre el uso de internet), Escala de Cohesión y Adaptabilidad Familiar y el Test de Adicción a Internet.

Cuestionario de datos sociodemográficos y de uso de internet.

Compuesta por dos secciones. La primera sección recoge información del sexo, edad, grado que cursaba, religión y procedencia. La segunda sección recoge datos sobre la familia y el uso de internet a través de tres preguntas con alternativas, tales como estructura familiar, tipo de uso de internet y lugar de conexión.

\section{Escala de Cohesión y Adaptabilidad Familiar}

La Family Adaptability and Cohesion Evaluation Scale (FACES III) fue elaborado por Olson, Portner y Lavee (1985). Está constituido por dos dimensiones: cohesión y adaptabilidad familiar. Contiene 20 ítems con una escala de respuestas de tipo Likert que van desde "Nunca" hasta "Siempre” y con una puntuación de 1 a 5 puntos respectivamente. Los 10 ítems impares corresponden a la dimensión de cohesión y los otros 10 ítems pares corresponden a la dimensión adaptabilidad (Polaino-Lorente y Martínez, 2003). Rosa María Reusche Lari en 1994 realizó la adaptación al Perú. La confiabilidad, determinada por test- retest, arrojó para cohesión 0.83 y para adaptabilidad 0.80 ; lo cual indica que el FACES III es confiable (Ferreira, 2003, Zambrano, 2011).

\section{Test de Adicción a Internet}

El Internet Addiction Test (IAT) fue creado por Young (1998b). Contiene 20 preguntas con una escala de respuesta tipo Likert ( 0 no aplica a $5=$ siempre), además tiene un rango de puntuación total de 20 a 100. Según las directrices de la autora los participantes que obtienen entre 20 y 49 puntos son usuarios con un control sobre el uso de internet, los que puntúan entre 50 y 79 tienen uso problemático del internet y los que puntúan más de 80 tienen problemas significativos, presentan uso adictivo de internet.

Puertas-Cortés y Carbonell (2013) realizaron la traducción y adaptación de la versión original del IAT al medio latino. Posteriormente, PuertasCortés, Carbonell y Chamarro (2013) realizaron un análisis de las propiedades psicométricas de la versión en español del IAT vía online, en una muestra de internautas colombianos, obtuvo un $\alpha=0.89$. Para esta investigación se utilizó el Alfa de Cronbach, con un resultado $\alpha=0.88$, la cual es indicador de una elevada fiabilidad. Asimismo la validez de contenido se obtuvo mediante el criterio de expertos. El grupo de jueces estuvo conformada por: un médico psiquiatra y dos psicólogos especialistas en adicciones, un psicólogo clínico especialista en diagnóstico y una psicóloga especialista en docencia e investigación. Los ítems 3, 11 y 15 presentaron observaciones por parte de uno o más jueces en cuanto a la claridad de los ítems; se hicieron las correcciones respectivas. 


\section{RESULTADOS}

Tabla 1

Estructura familiar y uso de internet de los participantes.

\begin{tabular}{cccc}
\hline Variables & Categorías & $\mathrm{n}$ & $\%$ \\
\hline \multirow{3}{*}{ Estructura familiar } & Nuclear & 93 & 41 \\
& Monoparental & 71 & 32 \\
Tipo de uso de internet & Extensa & 37 & 16 \\
& Reconstituida & 24 & 11 \\
& Redes sociales & 113 & 50 \\
& Juegos en red & 42 & 19 \\
& Vuscar información & 48 & 21 \\
& online (películas, series, etc.) & 16 & 8 \\
& Videos para adultos & 3 & 1 \\
Lugar de conexión a internet & Otros & 3 & 1 \\
& Domicilio & 85 & 38 \\
& Centro de estudios & 5 & 2 \\
& Cabina de internet & 76 & 34 \\
& Teléfono móvil & 56 & 25 \\
\hline
\end{tabular}

El 41\% pertenece a una familia nuclear y el 32\% a una incompleta. El 50\% utiliza el internet para las redes sociales, el $21 \%$ busca información y un $19 \%$ para juegos en red. El 38\% se conecta a internet desde su domicilio, seguido de un 34\% lo hace de una cabina de internet y un 25\% lo hace desde su teléfono móvil (ver tabla 2).

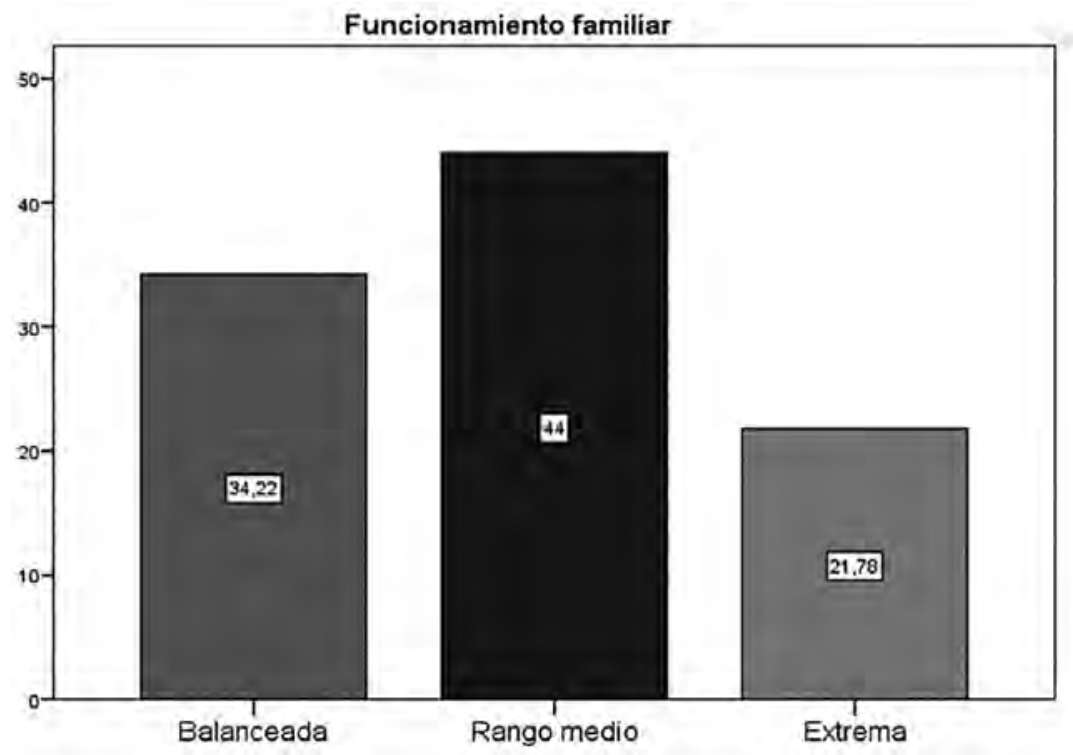

Figura 1

Niveles de funcionamiento familiar de los participantes.

En la figura 1 se visualiza que la mayoría de los adolescentes presenta un funcionamiento familiar de rango medio (44\%), solo un $21.7 \%$ tiene un funcionamiento extremo. 


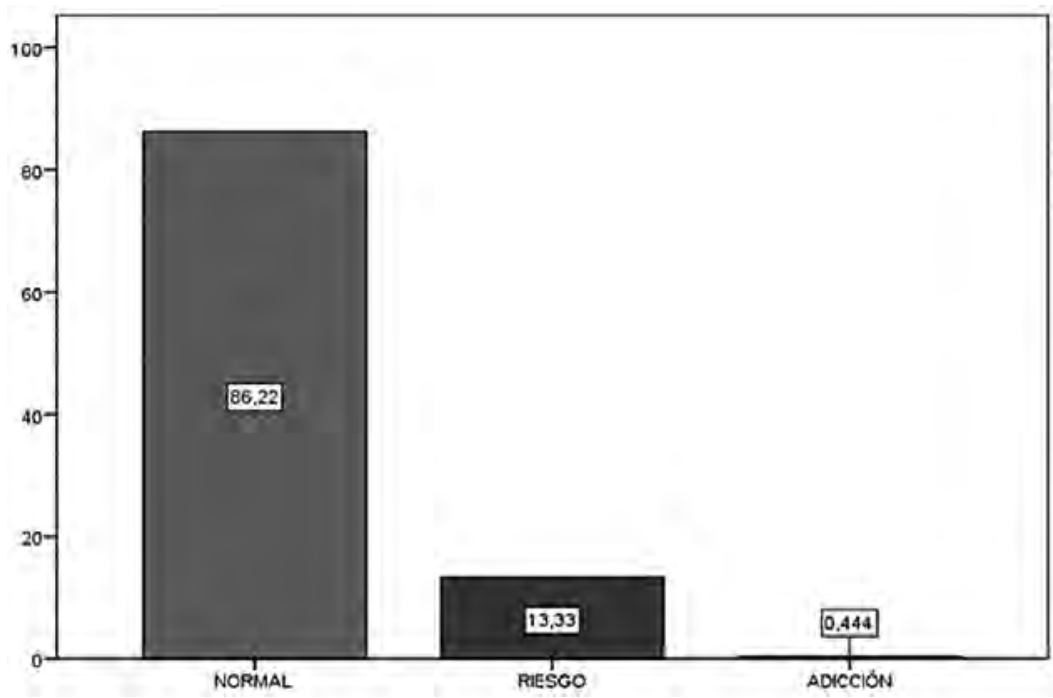

Figura 2

Niveles de adicción a internet en los participantes.

Asimismo, en la figura 2 se puede visualizar que el $86 \%$ de los participantes evidencia un uso normal de internet, el $13 \%$ se encuentran en riesgo de adicción y solamente cerca del $1 \%$ presenta un uso adictivo a internet.

Tabla 3

Análisis de correlación entre las variables de estudio.

\begin{tabular}{llll}
\hline & Valor & gl & p \\
\hline Chi-cuadrado de Pearson & 4,079 & 4 &, 395 \\
$\mathrm{~N}$ casos válidos & 225 & & \\
\hline
\end{tabular}

En la tabla 3 se puede apreciar en el análisis de correlación con la prueba estadística Chi-cuadrada que no existe relación significativa entre funcionamiento familiar y adicción a internet.

Tabla 4

Análisis de correlación entre las dimensiones de las variables de estudio.

\begin{tabular}{ccc}
\hline \multirow{2}{*}{ Funcionamiento Familiar } & \multicolumn{3}{c}{ Adicción a internet } \\
\cline { 2 - 3 } & $r$ & $\mathrm{p}$ \\
\hline Cohesión & $-.223^{* *}$ & .001 \\
Adaptabilidad & -.112 & .094 \\
\hline La correlación es significativa al nivel 0.01 (bilateral)
\end{tabular}

** La correlación es significativa al nivel 0.01 (bilateral)

En la tabla 4 se aprecia en el análisis de correlación con la prueba estadística $r$ de Pearson entre las dimensiones de la variable funcionamiento familiar y la adición a internet, se encontró que existe correlación significativa inversa $(\mathrm{r}=-.223, \mathrm{p}<0.01)$ entre cohesión familiar y adicción a internet, mientras que no se halló correlación significativa con adaptabilidad familiar $(\mathrm{r}=$ -.112, $\mathrm{p}<0.094)$. 


\section{DISCUSIÓN}

En esta investigación, se encontró que el funcionamiento familiar, siguiendo el modelo de Olson, no se relaciona significativamente con la adicción a internet. El funcionamiento familiar evaluado desde la percepción del alumno, mostró que independientemente sea el nivel de funcionamiento familiar, el adolescente puede adquirir o no la adicción a internet. Es importante hacer notar que estos resultados podrían indicar la presencia de otras variables intervinientes, tales como factores individuales como las características de la personalidad (impulsividad, timidez, introversión), baja autoestima o escasas habilidades sociales; así como el factor social (presión social) que intervienen en el riesgo de la adicción a internet (Echeburúa y Fernández-Montalvo, 2006; Echeburúa et al. 2009; Muñoz-Rivas et al. 2011).

En cuanto a la dimensión cohesión familiar, se encontró que existe relación significativa inversa $(\mathrm{r}=$ $-.223, \mathrm{p}<0.01)$ con la adicción a internet; es decir a mayor cohesión familiar (conectada y amalgamada), menor es el riesgo de los adolescentes de padecer adicción a internet. La cohesión familiar conectada se caracteriza por: cercanía emocional entre los miembros de la familia, límites generacionales claros, compartir amigos personales con la familia, comunicar las decisiones individuales y las importantes son hechas en familias, pasar tiempo juntos en familia involucrándose en actividades recreativas compartidas y también en intereses individuales, respetar el tiempo privado de los miembros por razones importantes. Esto se hace evidente a través de expresiones como: "los miembros de mi familia se piden ayuda unos a otros", "a los miembros de mi familia les gusta pasar su tiempo libre juntos" y "los miembros de mi familia se consultan entre sí sus decisiones”. Por lo contrario, la cohesión familiar amalgamada o aglutinada se caracteriza por: alta dependencia entre los miembros de la familia, poca individualidad, muchísimo tiempo juntos y los intereses y actividades tienen que ser con la participación familiar. En ambos casos existe relación con la adicción a internet, aunque una lo hace de manera funcional y la otra de forma disfuncional.

Entonces es razonable pensar que una menor cohesión familiar (separada y desligada) lleve al adolescente a buscar otros grupos de integración, focalizando sus actividades e intereses fuera de la familia, siendo vulnerable a conductas adictivas, entre ellas el internet; ya que está ofrece recompensas inmediatas, más aún cuando el objeto de la adicción está a su alcance (Echeburúa y Fernández-Montalvo, 2006). Asimismo Echeburúa et al. (2009) sustentan que una cohesión familiar débil es uno de los factores psicológicos que predisponen la adicción a internet en los adolescentes.

Este resultado se asemeja a lo encontrado por Alizadeh, Khosravi y Yusefhejad (2011); aunque no utiliza el modelo de funcionamiento familiar de Olson, las dimensiones que se relacionan negativamente (asertividad, orientación y entretenimiento) hacen referencia a la cohesión familiar de Olson. Asimismo Gunuc y Dogan (2013) encontraron una relación negativa $(r=-.37, \mathrm{p}<0.05)$ entre el apoyo social percibido y la adicción a internet; es decir, los adolescentes que pasan tiempo con sus padres compartiendo una serie de actividades (ver televisión, comer comidas, charlar, ir de compras y pasar tiempo fuera) tienen un menor nivel de adicción a internet. Aunque no utilizan el término funcionamiento familiar, sus actividades que comparten en familia son propias de una funcionalidad familiar. Por último, Vallejos y Capa (2010) también encontraron una relación inversa $(\beta=-.256, p<0.01)$ entre funcionamiento familiar y el uso del video juego, entendiéndose uso de video juego como el uso de juegos en red generando una adicción sin sustancia química. De esta manera los resultados encontrados por estos investigadores coinciden con lo encontrado por esta investigación.

Respecto a la dimensión adaptabilidad familiar se encontró que no existe relación significativa con la adicción a internet $(\mathrm{r}=-.112, \mathrm{p}>0,05)$. Estos resultados son contrarios a los encontrados por Wen Li, Garland y Howard (2014) donde al realizar una revisión sistemática de la adicción a internet en diversas investigaciones encontraron que los jóvenes adictos a internet reportan familias menos organizadas, cohesionadas y adaptadas. Donde las familias menos organizadas corresponderían según Olson a familias del tipo caóticas (extremo de la adaptabilidad), entendiéndose a ellas como aquellas familias donde existe falta de claridad en los roles y liderazgo, así como en el cambio frecuente de las reglas (PolainoLorente y Martínez, 2003).

No obstante es menester conocer que tales resultados provienen de una población muy distante al contexto latinoamericano, con características posiblemente distintas, viéndose necesario tomar en cuenta poblaciones que se asemejen a nuestro contexto sociodemográfico, debido a que tales resultados no se pueden ser generalizados para nuestra población nacional.

Probablemente la explicación se deba a la naturaleza misma de la dimensión adaptabilidad, la cual de acuerdo al modelo circumplejo de Olson es definida como la habilidad que tiene la familia para 
cambiar su estructura de poder, roles y las reglas de la relación en respuesta a estresores situacionales o evolutivos (Hidalgo y Carrasco, 1999); donde tales indicadores se ciñen exclusivamente a esta matriz conceptual.

De esta manera, si se hace un análisis introspectivo de los indicadores de esta dimensión se puede observar que los tales encuentran una mejor asociación con otras variables de investigación distintas a la adicción a internet. Tal como lo indican Pavez, Santander, Carranza y Vera-Villarroel (2009) al encontrar una asociación positiva entre la adaptabilidad familiar y la ideación suicida, es decir que un mayor nivel de adaptabilidad familiar resulta ser un factor de riesgo para la ideación suicida. Estos resultados son corroborados de cierta manera por Guibert y Torres (2001), quienes encontraron una tendencia a la poca adaptabilidad de las familias de individuos con intento suicida.

Desde el modelo circunflejo de Olson estos sistemas familiares operarían desde una forma muy rígida o muy flexible para cambiar su estructura de poder, relaciones de roles y reglas, ausencia o fuerte control parental y una disciplina poco efectiva (Ferreira, 2003), lo cual conllevaría según Almonte, Montt y Correa (citado por Pavez et al. 2009) a consecuencias negativas en la esfera emocional del adolescente.

Por su parte Ramírez (2007) encuentra bajos niveles de adaptabilidad en las familias (familias rígidas) con hijos drogodependientes. Rausch y Bay (1997) refieren que es común encontrar familias con tendencia a la rigidez y aglutinamiento en adolescentes anoréxicas. Asimismo Leyva, Hernández, Nava y López (2007) observaron que los adolescentes con depresión perciben a sus familias rígidamente dispersas. Estos alcances revelan la existencia de otras variables que tienden a relacionarse significativamente con la dimensión adaptabilidad familiar.

Se concluye que el funcionamiento familiar no se relaciona significativamente con el nivel de adicción de internet en los adolescentes de una institución educativa pública de Lima norte. Sin embargo, cabe señalar que se encontró relación negativa significativa con la dimensión cohesión familiar; esto quiere decir que cuanto mayor es la cohesión familiar, menor es el riesgo de adicción a internet en los adolescentes

\section{Declaración de financiamiento y de conflicto de intereses:}

El estudio fue financiado por los autores, quienes declaran no tener algún tipo de conflicto de interés en la investigación realizada.

\section{Correspondencia:}

\section{Cristian Adriano Rengifo}

Psicólogo Centro de Atención Psicológica, Universidad Peruana Unión. Carretera Central Km. 19.5 Ñaña, Lima, Perú.

e-mail: adrianorengifo12@gmail.com 


\section{REFERENCIAS BIBLIOGRÁFICAS}

Alizadeh, O., Khosravi, Z. y Yusefhejad, M. (2011). The relationship of internet addiction with family functioning and mental Health among Iranian students. European Psychiatry, 26(1), 18-22. Recuperado de http://www.sciencedirect.com/science/article/pii/ S092493381173526X

Arenas, S. (2009). Relación entre la funcionalidad familiar y la depresión en adolescentes. Tesis de licenciatura Universidad Nacional Mayor de San Marcos, Lima. Perú.

Banco Mundial. (2014). Recuperado de http://datos. bancomundial.org/indicador/IT.NET.USER.P2

Camelo, L., León, A. y Salcedo, C. (2013). Adicción a Internet: Aproximación a una perspectiva latinoamericana desde una revisión bibliográfica. Tercer Milenio, 18(25), 31-38. Recuperado de http://www.periodismoucn.cl/ tercermilenio/wp-content/uploads/Adicci\%C3\%B3na-Internet.-Aproximaci\%C3\%B3n-a-una-PerspectivaLatinoamericana-desde-una-Revisi\%C3\%B3nBibliogr\%C3\%A1fica.pdf

Cruzado, L., Matos, L. y Kendall, R. (2006). Adicción a Internet: Perfil clínico y epidemiología de pacientes hospitalizados en un instituto nacional de salud mental. Revista Médica Herediana, 17(4), 196-205. Recuperado de http://www.scielo.org.pe/pdf/rmh/v17n4/v17n4ao2. pdf.

Echeburúa, E. y Fernández-Montalvo, J. (2006). Tratado SET de trastornos adictivos. Cap. Adicciones sin drogas. Madrid: Editorial Médica Panamericana.

Echeburúa, E., Labrador, F. y Becoña, E. (2009). Adicción a las nuevas tecnologías en adolescentes y jóvenes. Madrid: Pirámide.

Ferreira, A. (2003). Sistema de interacción familiar asociado a la autoestima de menores en situación de abandono moral o prostitución. Tesis de Doctorado, Universidad Nacional Mayor de San Marcos, Lima. Perú. Recuperado de http://cybertesis.unmsm.edu.pe/ bitstream/cybertesis/559/1/ferreira_ra.pdf

Fontes, S., García, C., Quintanilla, L., Rodríguez, R., Rubio, P. y Sarriá, E. (2010). Fundamentos de investigación en psicología. España: UNED.

Guibert, W. \& Torres, N. (2001). Intento suicida y funcionamiento familiar. Revista Cubana Medicina General Integral, 17(5), 452-460. Recuperado de http://scielo.sld.cu/scielo.php?script=sci arttext\&pid=S0864-21252001000500008

Gunuc, S. \& Dogan, A. (2013). The relationships between Turkish adolescents' Internet addiction, their perceived social support and family activities. Computers in Human Behavior, 29(6), 2197-2207. Recuperado de http://www.sciencedirect.com/science/article/pii/ S0747563213001143

Hidalgo, C., y Carrasco, E. (1999). Salud Familiar. Un modelo de atención integral en la atención primaria. Chile: Imprenta Salesianos.

La Prensa (2014). Recuperado de http://laprensa.pe/ tecnologia-ciencia/noticia-adiccion-internet-chinatratamiento-19342
Leyva, R., Hernández, A., Nava, G. y López, V. (2007). Depresión en adolescentes y funcionamiento familiar. Revista Instituto Mexicano del Seguro Social, 45 (3), 225-232. Recuperado de http://cybertesis.unmsm.edu. pe/bitstream/cybertesis/2562/1/arenas_as.pdf

Luque, L. (2009). Uso abusivo y patológico de las tecnologías. Estudio descriptivo en jóvenes argentinos. Revista psicología.com, 12(2). Recuperado de http:// www.psiquiatria.com/revistas/index.php/psicologiacom/ article/viewFile/719/694

Matalinares, M., Díaz, G., Raymundo, O., Baca, D., Fernández, E., Uceda, J., Leyva, V., Sánchez, E., Villavicencio, N., Yaringaño, J., Torre, J., Encalada, M. y Díaz, A. (2013). Influencia de los estilos parentales en la adicción al Internet en alumnos de secundaria del Perú. Revista de Investigación en Psicología, 16(2), 195-220. Recuperado de file://E:/Tesis\%20\%202014/Estilos\%20 parentales\%20y\%20adiccion\%20a\%20internet.pdf

Morales, L. (2013). Factores de riesgo asociados a la conducta de adicción a internet en adolescentes de la I. E. Jorge Martorell Flores. Tesis de Licenciatura, Universidad Nacional Jorge Basadre Grohmann, Tacna. Perú. Recuperado de http://tesis.unjbg.edu.pe:8080/bitstream/ handle/unjbg/185/70_2013_Morales_Gallegos_LDC_ FACS_Enfermeria_2013.pdf?sequence $=1$

Muñoz-Rivas, M., Redondo, N., Fernández, L. y GámezGuadix, M. (2011). Manual de psicopatología y trastornos psicológicos. Cap. Las nuevas adicciones. España: Ediciones Pirámides.

Pavez, P., Santander, N., Carranza, J. y Vera-Villarroel, P. (2009). Factores de riesgo familiares asociados a la conducta suicida en adolescentes con trastorno depresivo. Revista Médica de Chile, 137, 226-233. Recuperado de http://www.scielo.cl/scielo.php?pid=S003498872009000200006\&script=sci_arttext

Polaino-Lorente, A. y Martínez, P. (2003). Evaluación psicológica y psicopatológica de la familia. España. Ediciones RIALP.

Puertas-Cortés, D. y Carbonell, X. (2013). Uso problemático de internet en una muestra de estudiantes universitarios colombianos. Avances en Psicología Latinoamericana, 31(3), 620-631. Recuperado de http://www.scielo.org. co/pdf/apl/v31n3/v31n3a12.pdf

Puertas-Cortés, D. \& Carbonell, X. y Chamarro, A. (2013). Análisis de las propiedades psicométricas de la versión en español del Internet Addiction Test. Tratarnos Adictivos, 14(4), 99-104. Recuperado de http://www. academia.edu/4764020/Analisis_de_las_propiedades_ psicometricas_de_la_version_en_espanol_del_Internet_ Addiction_Test

Ramírez, L. (2007). El funcionamiento familiar en familias con hijos drogodependientes. Un análisis etnográfico. Tesis doctoral. Universidad de Valencia, Valencia. España. Recuperado de http://hdl.handle. net/10803/10191

Rausch, C. y Bay, L. (1997). Anorexia Nerviosa y Bulimia: amenazas a la autonomía. Buenos aires: Paidós. 
Toro, J. (2010). El adolescente en su mundo. Riesgos, problema y trastornos. Madrid: Pirámide.

Universia (2010). Recuperado de http://noticias.universia. net.mx/en-portada/noticia/2010/12/30/775606/adictosinternet-2-jovenes-mexicanos.html

Vallejo, M. \& Capa, W. (2010). Video juegos: adicción y factores predictores. Revista Unife, 18(1), 103110. Recuperado de http://www.unife.edu.pe/pub/ revpsicologia/miguelvallejos.pdf.

Wen Li, Garland, E. y Howard, M. (2014). Family factors in Internet addiction among Chinese youth: A review of English- and Chinese-language studies. Computers in Human Behavior, 31(1), 393-411. Recuperado de http://www.sciencedirect.com/science/article/pii/ S074756321300424X

Young, K. (1998b). Internet addiction: the emergence of a new clinical disorder. Cyber psychology and Behavior, 1(3), 237-244. Recuperado de http://netaddiction. fusionxhost.com/articles/newdisorder.pdf
Zambrano, A. (2011). Cohesión, adaptabilidad familiar y el rendimiento académico en comunicación de alumnos de una Institución Educativa del Callao. Tesis de Maestría. Universidad San Ignacio De Loyola, Lima. Perú. Recuperado de http://repositorio.usil.edu.pe/wp-content/ uploads/2014/07/2011_Zambrano_Cohesi\%C3\%B3na daptabilidad-familiar-y-rendimientoacad\%C3\%A9mico-en-comunicaci\%C3\%B3n-dealumnos-de-una-instituci\%C3\%B3n-educativa-delCallao.pdf

Zapata, G. (2013). Uso problemático de internet en adolescentes atendidos en consulta externa del Hospital Hermilio Valdizán en el periodo 20092011. Tesis inédita de Especialista en Psiquiatría. Universidad Nacional Mayor de San Marcos, Lima. Perú. Recuperado de http://ateneo.unmsm.edu.pe/ ateneo/bitstream/123456789/2971/1/Zapata_Coacalla_ Giovanna_2013.pdf

Recibido: 19/05/2015 Aceptado: 28/10/2015 